\title{
GSA Application in Energy Management
}

\author{
Zhang Wenyu ${ }^{1, a}$, Xue $\mathrm{Yu}^{2, \mathrm{~b}}$,Yang Fengxia ${ }^{1, \mathrm{c}}, Y u$ Qi ${ }^{1, \mathrm{~d}}$ and Fan Haiyan ${ }^{1, \mathrm{e}}$ \\ ${ }^{1}$ School of Economics and Management, Xi' an University of Posts and Telecommunications, Xi' an, \\ 710061 \\ ${ }^{2}$ Second Academy of China Aerospace Science \& Industry Corporation, Beijing, 100854

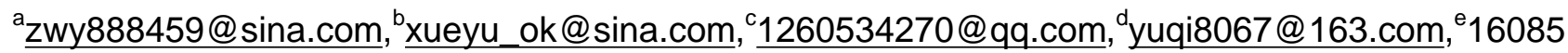 \\ 57213@qq.com
}

Keywords: Energy management; Grey system; Grey prediction; Grey correlation analysis

Abstract: Energy consumption has seen a sharp increase with the rapid sci-tech development in China, which leads to an urgent demand on efficient, scientific and reasonable energy management methods to predict and evaluate energy consumption and improve the energy utilization rate. Based on the theory of grey system analysis (GSA), two methods, the grey prediction and grey correlation analysis, are applied in the paper to the prediction and evaluation of energy consumption, which is the main focus of energy management. Following it, the validity and practicability of models constructed in the paper are tested through a empirical study of the relevant data of a residential project in Gaoling county. The scientific prediction and evaluation of energy consumption is realized in this research to provide scientific decision-making basis for the energy savings of the project units, and a strong theoretical and guiding significance for the development of energy management in the future.

\section{Introduction}

Energy is an important material basis for economic development and social progress. In the middle and late 20th century, the scarcity of global resources and the shortage of energy reserves have become the main factor restricting the economic development of all countries. To realize the rapid and all-round economic development, it is of an inevitable step to carry out resource saving projects, and speed up the construction of a resource-saving society, which is also the only way to implementing a comprehensive coordinated sustainable development and promoting the harmonious development between man and nature.

While promoting economic development, energy exploitation in China has unfortunately led to a series of problems threatening economic and social sustainable development. Faced with such a grim energy situation, on the one hand, China's energy enterprises should follow the idea of modest leading development; on the other hand, they should improve energy efficiency, strengthen awareness of environmental protection, and always make the construction of resource-saving, environmentally friendly society as the goal of China's energy industry development. The major problems of energy use in China are inefficiency and serious waste due to enterprises' insufficient awareness of the importance and urgency of energy management. Hence only by arousing the awareness of efficient and fine management of the energy management system, can scientific energy management be realized? To achieve effective energy management, the control and supervision of its whole process including the standardization management of energy, energy-saving target management, energy prediction, energy metering and energy evaluation must be carried out. As a result, the formulation of reasonable energy management methods and the 
adoption of appropriate energy management solutions have become the priority to solve energy problems.

\section{Energy Management and Grey System Overview}

Energy Management

Concept of Energy Management

The content of this paper is based on the broad sense of energy management, which is defined as the management of energy production process and consumption process ${ }^{[1]}$. The main content of energy management is accurate monitoring and scientific analysis of energy consumption. Based on it, the corresponding management and technical measures are taken to achieve the goal of energy savings.

Functions and Basic Work of Energy Management

Energy management is an important part of the energy savings, and its specific functions are as follows ${ }^{[2]}$ :Planning function, Organizing function, Commanding function, Coordinating function, Controlling function. The above-mentioned energy management functions are interrelated and restricting each other. Under different conditions, different energy management levels focus on different aspects. Thus, these functions should be organically combined during implementation in view of the actual situation.

The basic work of energy management refers to the rational and effective management of all the links of energy use in order to reduce energy loss and improve the energy efficiency. It includes five aspects ${ }^{[3]}$ :

(1)Standardization of Energy Management ; (2) objective management of energy savings;(3) Energy prediction;(4) Energy metering;(5) Energy evaluation. The basic work of energy management is shown in Figure 1:

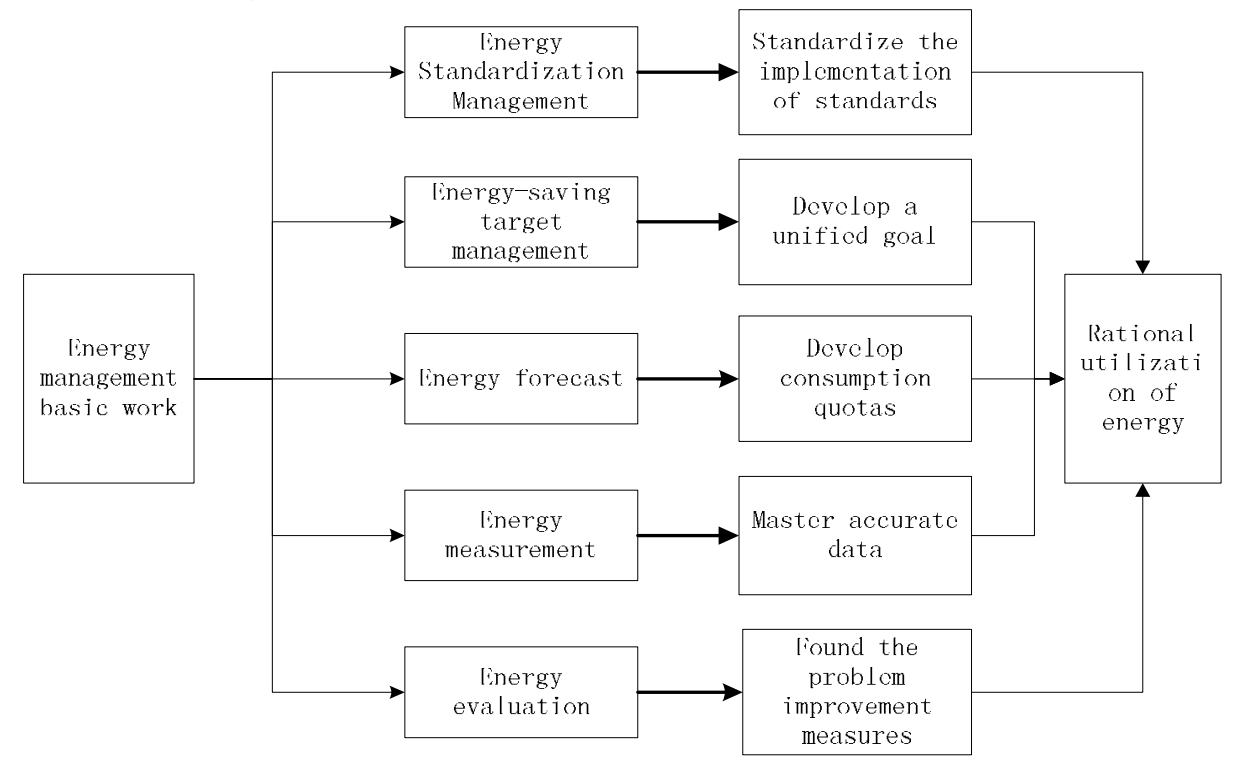

Fig. 1 schematic diagram of energy management basic work 


\section{Grey System Overview}

Grey System Concepts and Tasks

Grey System, proposed by Deng Julong, a professor and a doctoral adviser of Huazhong University of Science and Engineering in 1982, is a mathematical method to solve the incomplete information system. It extends the viewpoint and method of cybernetics into the complex giant system, combines the automatic control with the mathematical method of operational research, and uses the unique methods and means to study the grey problem in the objective world ${ }^{[4]}$.

The research process of the grey system theory involves the black box with completely unknown internal structure and features, the grey box with partially unknown structure and features, and the grey system.

It is known that the grey system theory began to be applied to agriculture and other fields and has achieved good results ${ }^{[5]}$. Focusing on social and economic research, the grey system theory will play an increasingly important role in economic planning, natural resource development and future prediction in that the main research tasks of GSA are data analysis, mathematical modeling, development forecasting, decision evaluation, overall planning, global control and etc. for all kinds of grey systems such as energy consumption, social and economic development, and ecological environment.

\section{Basic Methods of GSA}

The GSA theory consists of three kinds of systems, the grey one, the white one and the black one, with the latter two as the special cases. Through an in-depth analysis and comprehensive understanding of the grey system, the GSA theory has formed its own unique research and analysis methods.

\section{(1) Grey prediction}

In the study of uncertain problems with insufficient data and poor information, the grey system theory has its unique effect and role, because "small sample and poor information" problems with "partially known information" are the research objects of the grey system theory, which can extract the valuable information from the initial generation and later development of the known information in the system, and realize the correct description and effective monitoring in the system operation and development. As one of the most important contents in the grey system theory and a new branch of the prediction theory, the grey prediction technology, aiming at grey uncertainty prediction problems abundantly existing in the real world, makes use of a small amount of valid data and grey uncertain data to reveal the future development trend of the system through the cumulative generation of sequences ${ }^{[6]}$.

\section{(2) Grey correlation analysis}

Uncertain correlations among things, among system factors, or among principal factors and subordinate factors are called grey correlations. Grey correlation analysis is to analyze and determine the impact degree of the system factors or the contributions of the main behavior of the system by using the grey correlation sequence (GCS) to describe the extent and sequence of relations among the factors. As the basis of analysis, evaluation and decision-making of the grey system, correlation analysis focuses on measuring the correlation between factors, according to the dissimilarity degree and similarity degree of the development situation between factors, which is an effective, objective and practical mathematical method. Among various correlation models, typical ones are Deng's Correlation, Generalized Absolute Correlation, T-type Correlation, Grey Slope 
Correlation, B-type Correlation and Improved Correlation.

\section{Construction of Energy Management Model Based on GSA}

Energy prediction modeling based on grey prediction

As the basis of the grey system prediction, $\operatorname{GM}(1,1)$ model, consisting of first-order differential equation with a single variable, is an effective model of energy demand prediction, and a special case of $\operatorname{GM}(1,1)$ model. So, $\operatorname{GM}(1,1)$ algorithm will be applied in the energy prediction process . The specific modeling process is as follows.

(1) Raw data pre-processing

Raw data are collected in accordance with the indexes requiring prediction. In this case monthly electricity consumption data of the Gaoling residential project is used.

non-negative original sequences:

$$
X^{(0)}=\left\{x^{(0)}(1), x^{(0)}(2), \cdots, x^{(0)}(n)\right\}
$$

$x^{(0)}(1), x^{(0)}(2), \cdots, x^{(0)}(n)$ represents the monthly power consumption and $\mathrm{n}$ represents the number of months. The resulting sequence is obtained through an accumulation of 1-AGO:

$$
X^{(1)}=\left\{x^{(1)}(1), x^{(1)}(2), \cdots, x^{(1)}(n)\right\}
$$

In the

$$
x^{(1)}(k)=\sum_{i=1}^{k} x^{(0)}(i) .
$$

The first order linear differential equation of power consumption is constructed.

$$
\frac{d x^{(1)}}{d t}+\alpha x^{(1)}=u
$$

(2) Prediction model

Based on the linear differential equation of power consumption, the least squares method is used to solve the parameter and U.

$$
\left[\begin{array}{l}
\hat{\alpha} \\
\hat{u}
\end{array}\right]=\left(B^{T} B\right)^{-1} B^{T} Y_{n} .
$$

In the

$$
B=\left[\begin{array}{cc}
-\frac{1}{2}\left[x_{1}^{(1)}(1)+x_{1}^{(1)}(2)\right] & 1 \\
-\frac{1}{2}\left[x_{1}^{(1)}(2)+x_{1}^{(1)}(3)\right] & 1 \\
\mathrm{M} & \mathrm{M} \\
-\frac{1}{2}\left[x_{1}^{(1)}(n-1)+x_{1}^{(1)}(n)\right] & 1
\end{array}\right], Y_{n}=\left[\begin{array}{c}
x^{(0)}(2) \\
x^{(0)}(3) \\
\mathrm{M} \\
x^{(0)}(n)
\end{array}\right] .
$$

Returning $\hat{\alpha}, \hat{u}$ to the original differential equation, it can be obtained:

$$
\hat{x}^{(1)}(k+1)=\left[x^{(1)}(1)-\frac{\hat{u}}{\hat{\alpha}}\right] e^{-\hat{\alpha} k}+\frac{\hat{u}}{\hat{\alpha}}(\mathrm{k}=1,2, \ldots) .
$$


After the reduction, the grey prediction model of the sequence $x^{(0)}$ is

$$
\hat{x}^{(0)}(k+1)=\left(e^{-\hat{\alpha}}-1\right)\left[x^{(0)}(1)-\frac{\hat{u}}{\hat{\alpha}}\right] e^{-\hat{\alpha} k},(k=1,2, \mathrm{~L})
$$

Formula (5) is the prediction model of power consumption of the residential project. Based on the prediction model, it is also necessary to determine the scope of the model and determine whether the model is suitable for prediction. All the prediction models have their application limits and $\operatorname{GM}(1,1)$ model is no exception. Confined to the development coefficient, application of the model in prediction is meaningful only under the conditions of $|\alpha|<2$. Moreover, with the increase of the development coefficient, the simulation error increases rapidly ${ }^{[7]}$.

(3) Posterior-variance-test of the model

$\hat{x}^{(1)}$ has been solved based on $\operatorname{GM}(1,1)$ Modeling method. A reduction operation is now done to get

$$
e(k)=x^{(0)}(k)-\hat{x}^{(0)}(k), k=1,2, \mathrm{~L}, n .
$$

The residual error is

$$
E=[e(1), e(2), \mathrm{L}, e(n)]=X^{(0)}-\hat{X}^{(0)} .
$$

At this time there are:

$$
\begin{aligned}
& S_{1}^{2}=\frac{1}{n} \sum_{k=1}^{n}\left[x^{(0)}(k)-\bar{x}\right]^{2} . \\
& S_{2}^{2}=\frac{1}{n} \sum_{k=1}^{n}[e(k)-\bar{e}]^{2} .
\end{aligned}
$$

Among them

$\bar{x}=\frac{1}{n} \sum_{k=1}^{n} x^{(0)}(k), \bar{e}=\frac{1}{n} \sum_{k=1}^{n} e(k) . S_{1}^{2}$ is the variance of the original sequence $X^{(0)} ; S_{2}^{2}$ is the variance of the residual sequence $\mathrm{E}$. The posterior error ratio is

$$
C=\frac{s_{2}}{s_{1}} .
$$

The probability of small error is $\quad p=p\left\{|e(k)-\bar{e}|<0.6745 S_{1}\right\}$.

The results of the model after the posterior-variance-test can be divided into four grades ${ }^{[8,9,10]}$ according to the range of index $\mathrm{C}$ and $\mathrm{P}$. The smaller Indicator $\mathrm{C}$ is better. A smaller $\mathrm{C}$ indicates a larger $s_{1}$, and a smaller $s_{2}$. A larger $s_{1}$ indicates a bigger variance and a bigger discrete degree of the historical data. A smaller $s_{2}$ shows a smaller residual variance and the residual difference. A small $\mathrm{C}$ indicates that the difference between the predicted value and the actual value of the model is not too discrete in spite of the discrete historical data. The larger the index $\mathrm{P}$, the better because a larger $\mathrm{P}$ shows that the difference between the residual and residual mean is further less than the given value. 
Energy evaluation model based on grey correlation analysis

(1) Determining index data matrix

According to the specific energy consumption index and the statistic energy consumption data of the Gaoling residential project, the data matrix of the index is built as follows :

$$
X=x_{i}(k)=\left|\begin{array}{cccc}
x_{1}(1) & x_{1}(2) & \mathrm{L} & x_{1}(n) \\
x_{2}(1) & x_{2}(2) & \mathrm{L} & x_{2}(n) \\
\mathrm{M} & \mathrm{M} & \mathrm{M} & \mathrm{M} \\
x_{m}(1) & x_{m}(2) & \mathrm{L} & x_{m}(n)
\end{array}\right| .
$$

In the formula, $x_{i}(k)$ represents the data value of the index $\mathrm{I}$ of the $\mathrm{K}$ object, $i \in[1, m], k \in[1, n]$, and $\mathrm{M}$ denotes the number of indicators and $\mathrm{N}$ indicates the number of objects evaluated. In the residential project, $\mathrm{M}$ represents the 12 months of the year and $\mathrm{N}$ is the evaluation targets, namely the main equipment power consumption, public lighting power consumption, residential power consumption, auxiliary facilities power consumption, residential and urban green belt water consumption, and natural gas.

(2) Determining the reference sequence

The principle of determining reference sequence is that data in the reference sequence is composed of the sum of optimal values of each index. The reference sequence $\left\{X_{0}\right\}$ is marked as $\left\{X_{0}\right\}=\left\{X_{0}(1), X_{0}(2), \mathrm{L}, X_{0}(n)\right\} \quad$ In the formula, $\mathrm{N}$ is the number of objects evaluated.

(3) Data pre-processing

Before the correlation analysis, the original data of the system shall be given a dimensionless treatment to achieve dimensional consistency because of the different data units or dimensions. Mean value method is hereby used as follows:

$$
X^{\prime}=\frac{x_{i}(k)}{\bar{x}(k)}, i \in[1, m], k \in[1, n] .
$$

Formula (12) $\bar{X}(k)$ represents the average of all the indicators of each subject, namely, the main equipment power consumption, public lighting power consumption, residential power consumption, auxiliary facilities power consumption, residential and urban green belt water consumption, and natural gas.

The dimensionless data matrix of the indicators (including reference data columns $\left\{X_{0}^{\prime}(i)\right\}$ can be obtained.

$$
X^{\prime}=x_{i}^{\prime}(k)=\left|\begin{array}{cccc}
\frac{x_{1}(1)}{\bar{X}(1)} & \frac{x_{1}(2)}{\bar{X}(2)} & \mathrm{L} & \frac{x_{1}(n)}{\bar{X}(n)} \\
\frac{x_{2}(1)}{\bar{X}(1)} & \frac{x_{2}(2)}{\bar{X}(2)} & \mathrm{L} & \frac{x_{2}(n)}{\bar{X}(n)} \\
\mathrm{M} & \mathrm{M} & \mathrm{M} & \mathrm{M} \\
\frac{x_{m}(1)}{\bar{X}(1)} & \frac{x_{m}(2)}{\bar{X}(2)} & \mathrm{L} & \frac{x_{m}(n)}{\bar{X}(n)}
\end{array}\right| .
$$

(4) Differential sequence and maximum and minimum values 
The absolute difference between the dimensionless value $X_{i}^{\prime}(k)$ and the reference sequence $X_{0}^{\prime}(i)$ of each data in the index system is calculated in the formula: $\Delta_{i}(k)=\left|X_{0}^{\prime}(i)-x_{i}^{\prime}(k)\right|$ $i \in[1, m], k \in[1, n]$, Here $\mathrm{m}$ indicates the number of index, and $\mathrm{n}$ represents the number of objects being evaluated.

The maximum and minimum values are determined as follows:

(1) The maximum value is :

$$
M=\max _{i} \max _{k} \Delta_{i}(k) \text {. }
$$

(2) The minimum value is:

$$
m=\min _{i} \min _{k} \Delta_{i}(k)
$$

$i \in[1, m], k \in[1, n]$, Here $\mathrm{m}$ indicates the number of indicators, and $\mathrm{N}$ represents the number of objects being evaluated.

(5) Calculation of correlation coefficient and correlation

In the process of modeling, Deng's correlation is used to calculate the specific correlation. The calculation formula for correlation coefficient is:

$$
\xi_{i}(k)=\frac{m+\rho M}{\Delta_{i}(k)+\rho M} .
$$

Here is the resolution coefficient, $\rho \in[0,1]$ generally, $\rho=0.5$.

The correlation is obtained by the calculation of the means of the correlation coefficients of each sequence.

\section{Application of GSA in Energy Management Process}

In this section, grey prediction and grey correlation analysis are applied to predict and evaluate the energy consumption of Gaoling residential project. The data used in the process is the tracking record of the residential project, and the results can serve as a solid guidance to the future energy savings of this project.

\section{Application of Grey Prediction Model in Energy Consumption Prediction}

According to the modeling process of grey prediction theory, $\operatorname{GM}(1,1)$ model is used in the prediction of monthly electricity consumption of the Gaoling residential project in the last four months of the year. In this case only the electricity power consumption is verified in spite of the prediction ability of other kinds of energy consumption. DPS data processing system is used to calculate the related data and the specific data and part of the calculation process are as follows [11,12,13,14]:

The power consumption of a residential project in the first 8 months of the year is shown in the following table: 
Table 1 First 8 months power consumption

meter unit: $10 \mathrm{k} \mathrm{kwh}$

\begin{tabular}{|c|c|c|c|c|c|c|c|c|}
\hline month & January & February & March & April & May & June & July & August \\
\hline power consumption & 133 & 134 & 135 & 136 & 135 & 137 & 138 & 137 \\
\hline
\end{tabular}

The parameters in the differential equation of $\mathrm{GM}(1,1)$ are obtained by calculating the cumulative generation value of the original sequence and the data matrix $\mathrm{B}$ and the data vector. $\hat{\alpha}=-0.0042, \hat{u}=133.4536$

So far, the grey prediction model of the cumulative sequence is as follows:

$$
\hat{x}^{(1)}(k+1)=31930 e^{0.0042 k}-31774.67
$$

After reduction of formula (4), the grey prediction model of the sequence is:

$$
\hat{x}^{(0)}(k+1)=31930\left(e^{0.0042}-1\right) e^{0.0042 k}
$$

Based on the above two models, it can be obtained that

Table 2 Model Value Calculation

\begin{tabular}{|c|c|c|c|c|c|c|c|c|}
\hline $\mathrm{K}$ & 1 & 2 & 3 & 4 & 5 & 6 & 7 & 8 \\
\hline$\hat{x}^{(1)}(k)$ & 289.7 & 425 & 560 & 692 & 833 & 970 & 1108 & 1246.37 \\
\hline$\hat{x}^{(0)}(k)$ & 134.56 & 135.13 & 135.69 & 136.27 & 136.84 & 137.41 & 137.99 & 138.56 \\
\hline
\end{tabular}

In order to ensure the reliability of the predictive model data, it is necessary to check the accuracy of the predictive model. After calculation, the error ratio is:

$$
C=\frac{S_{2}}{S_{1}}=0.405
$$

The probability of small error is: $P=P\left\{|e(k)-\bar{e}|<0.6745 S_{1}\right\}=0.84$.

The prediction accuracy of the model is good according to the posterior error ratio and the small error probability, so the power consumption of the remaining four months can be predicted. The specific values are as follows: $\hat{x}^{(0)}(k+1)=31930\left(e^{0.0042}-1\right) e^{0.0042 k}$

$$
\hat{x}^{(0)}(9)=138.29, \hat{x}^{(0)}(10)=138.87, \hat{x}^{(0)}(11)=139.46, \hat{x}^{(0)}(12)=140.05
$$

The prediction values indicate that the electricity consumption of the residential project in the late four months of the year is in a steady upward trend while a further observation is needed due to small monthly increase.

\section{Application of Grey Correlation Analysis Model in Energy Consumption Evaluation}

The prerequisite of a multi-index comprehensive evaluation of residential building energy savings is to determine the scientific evaluation index system, only with which it is possible to make a scientific and impartial evaluation on residential building energy savings. It means the application of statistical analysis method in analysis of the energy consumption of residential buildings, in order to adopt reasonable energy saving approaches and strengthen the efficiency of energy utilization by paying more attention to the key energy consumption in the future work. The energy consumption system of the residential project is illustrated in the following diagram: 


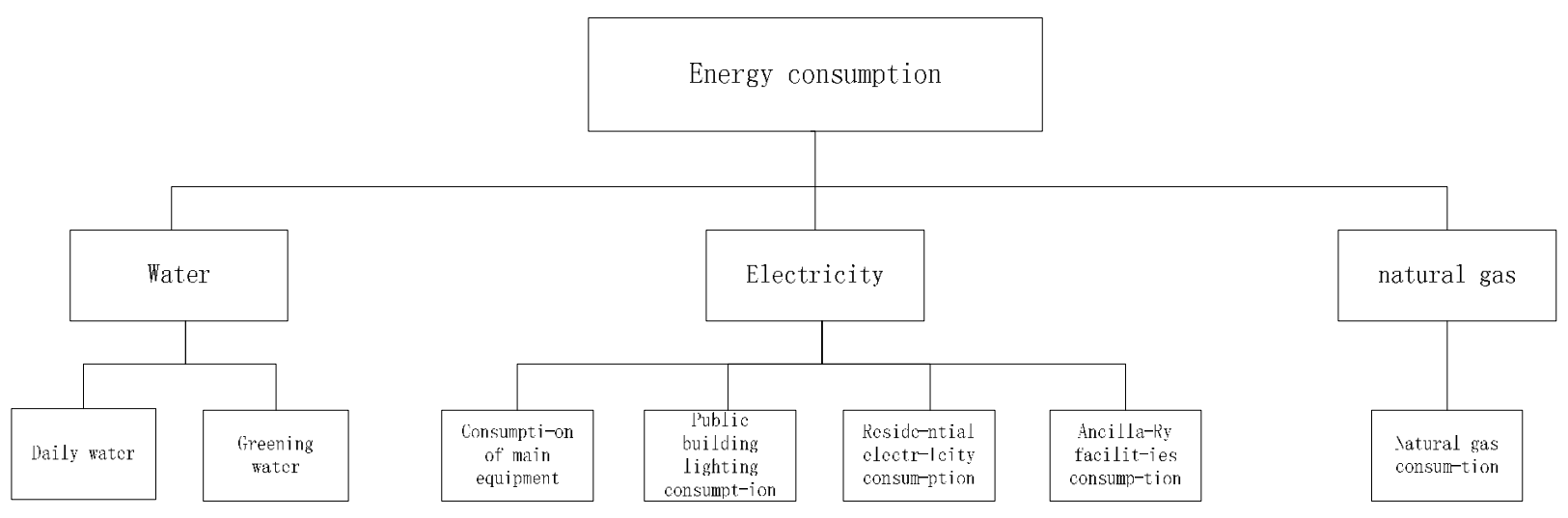

Fig. 2 Energy Consumption System of the Residential Project

After the conversion of energy consumption index coefficients to standard coal consumption and standardized treatment, according to the calculation formula in section 2.2, the difference sequence and the maximum and minimum values are computed and correlation coefficients of the energy consumption indexes, then correlation can be obtained by calculating the average of each sequence:

$\mathrm{R}$ (main equipment power consumption $)=(0.9433+0.8840+0.9575+0.9791+0.9531+0.9502+$

$0.9656+0.9886+0.9190+0.8906+0.9656+0.8846) / 12=0.9426$

Likewise,

$\mathrm{R}$ (Public lighting power consumption) $=0.4677$;

$\mathrm{R}$ (Residential power consumption) $=0.4742$;

$\mathrm{R}$ (Auxiliary facilities power consumption) $=0.4699$;

$\mathrm{R}$ (residential water consumption) $=0.4756$;

$\mathrm{R}$ (urban green belt water consumption) $=0.4578$;

$\mathrm{R}$ (Natural gas consumption) $=0.4762$.

Based on the correlation, we get:

$\mathrm{R}$ (The main equipment power consumption) $>\mathrm{R}$ (gas consumption) $>\mathrm{R}$ (domestic water) $>$

$\mathrm{R}($ Residential electricity consumption) $>\mathrm{R}$ (power consumption of auxiliary equipment) $>$

$\mathrm{R}$ (power consumption of Public building lighting) $>\mathrm{R}$ (water for afforestation)

The results show that the main equipment power consumption accounts for the largest proportion, followed by natural gas consumption and domestic water. To achieve the minimum energy consumption, therefore, equipment management and upgrading and inspection should be strengthened, high energy-consuming equipment in the replacing list of China should be replaced and regular check should be implemented. Meanwhile, natural gas consumption monitoring should be strengthened in order to make maximum use of it.

As a case study of grey prediction and grey correlation analysis, this section, following a prediction of electricity consumption based on the energy consumption data of Gaoling residential project, makes a grey correlation analysis of power consumption of various projects in the whole year to identify the key energy consumption projects, which points out the direction for the future energy savings and provides scientific basis for the future decision-making of energy saving managers. Specific energy saving measures of the project are presented at the end of the section.

\section{Conclusion}

Since the reform and opening up, China has done a lot of work in energy savings, especially the energy management. How to evaluate whether the energy consumption of a project conforms to 
the standard and whether the project requires an improvement in energy saving are currently the research focuses of energy savings in China. This paper focuses on the development background and basic concepts of energy management, and expounds its important role in China's economic development. In view of the specific work in the energy management process, the application of GSA in energy management is introduced in this paper. Then, the prediction and evaluation models are established with grey prediction and grey correlation analysis respectively and are tested based on the actual data of Gaoling residential building project. It turns out that the empirical results are consistent with the reality of the project, and have a strong theoretical and instructive significance for the development of energy management in the future.

\section{References:}

[1] Gong Huan. Enterprise energy management [M]. Shanghai Science Popularization Publishing House, 1999.

[2] Liang Zhaohui. Urban Energy Management: International Comparison and Empirical Study in China [M]. Shanghai People's Publishing House, 2010.

[3] Chen Zhitian. Energy management of the whole process of [M]. Beijing: China Standard Press, 2012.

[4] Li Yan. Gray prediction model research and its application [D]. Zhejiang University of Technology, 2012.

[5] Zhang Yongbo.Study on prediction model based on gray system theory [D]. Harbin Engineering University, 2005.

[6] Tao Ran. Shanghai can and forecast models and energy efficiency evaluation index system research [D]. Shanghai Jiaotong University, 2012.

[7] Zhao Ying. Energy forecast and energy optimization technology in metallurgical enterprises in the application of [D]. Changchun University of Technology, 2010.

[8] Burhan Ozkan, Handan Akcanz and CemalFert. Energy input-output analysis in Turkish agriculture [J]. Renewable Energy, 2004, 29: 39-511.

[9] Cheng Maolin. A New Approach to the Improvement of Gray GM (1,1) Model [J]. Journal of Statistics and Decision. 2004, (2): 16-17.

[10] Zhou Ruiping. Prediction of Urban Population by GM $(1,1)$ Model Gray Forecasting Method [J]. Journal of Inner Mongolia Normal University (Natural Science Edition). 2005,34 (1): 81-83.

[11] Tang Pingya. Electric demand forecasting method and energy demand forecasting model [J]. China Electric Power. 2000, 33 (4): 32-34.

[12] Guo Yuntao. China's coal long-term supply and demand analysis and forecast [J]. China Coal .2004,30 (10): 20-24.

[13] Chang K. C and YehM. F, Gray relational analysis based approach for data clustering, IEE Proceedings-Vision, Image \& Signal Processing, 2005, 152 (2): 165-172.

[14] AlcantaraVicent. Rosa Duarte .Comparison of energy intensities in European Union countries, results of a structural decomposition oil. Energy Policy. 2004, 32 (2): 177-189. 